\title{
Mediastinal teratoma with hydrops fetalis in a newborn and development of chronic respiratory insufficiency
}

\author{
Milanka Simoncic ${ }^{1}$, Silvo Kopriva1', Ziva Zupancic², Maja Jerse ${ }^{3}$, Janez Babnik ${ }^{4}$ \\ Matevz Srpcic ${ }^{5}$, Stefan Grosek ${ }^{1}$

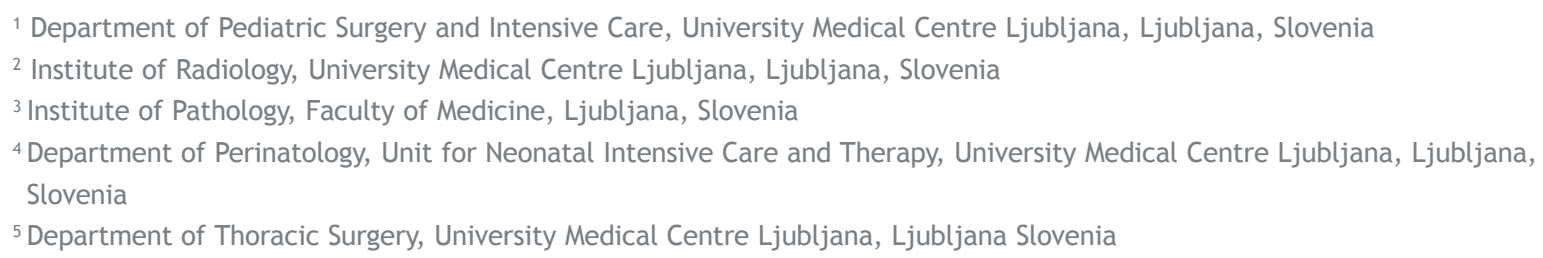

Radiol Oncol 2014; 48(4): 397-402.

Received 28 June 2013

Accepted 24 July 2013

Correspondence to: Prof. Stefan Grosek, M.D., Ph.D., Associate Professor in Paediatrics, Department of Pediatric Surgery and Intensive Care, University Medical Centre Ljubljana, Bohoriceva 20, 1525 Ljubljana, Slovenia. E-mail: stefan.grosek@kclj.si or stefan.grosek@mf.uni-lj.si

Disclosure: No potential conflicts of interest were disclosed.

Background. Mediastinal fetal teratoma can be detected as a mass in the chest during a routine prenatal ultrasound screening. Because of the pressure on mediastinal structures it can be the cause of non-immune hydrops fetalis and polyhydramnion. The development of hydrops fetalis leads to fetal death or premature delivery in most reported cases. Early surgical removal is important, but, the result of treatment depends on the stage of development of mediastinal organs and complications in the postoperative period.

Case report. A 31-year-old gravida carrying twins, with spontaneous membrane rupture at 32 weeks gestation underwent urgent caesarean section after antenatal ultrasound revealed severe polyhydramnion and hydrops fetalis in geminus A. The child was intubated immediately after birth due to severe respiratory distress. Ultrasound and X-ray revealed a tumour mass in the right hemithorax. Tumour resection was performed at the age of 7 days. Histology examination revealed an encapsulated immature teratoma. The postoperative course was complicated with respiratory insufficiency which turned into chronic at the age of eight months.

Conclusion. This is the fifth reported child with fetal mediastinal teratoma and severe hydrops fetalis that survived the neonatal period. Additional diagnostic search revealed abnormal course of both pulmonary arteries, which was probably one of the main causes of respiratory insufficiency.

Key words: mediastinal teratoma; non-immune hydrops fetalis; diaphragm paralysis; chronic respiratory insufficiency

\section{Introduction}

Mediastinal teratomas are the second most common extragonadal teratomas in children. ${ }^{1}$ There are some reports of mediastinal teratomas discovered as a mass in the chest with antenatal ultrasound (US) in fetal life..$^{2-8}$ Rapid growth in fetal life can compress lungs, heart and great vessels, which can lead to development of non-immune hydrops fetalis (NIHF). ${ }^{7}$ High mortality can be prevented only by an early intervention at the onset of NIHF that dramatically improves deteriorating condition and survival rate of fetuses. ${ }^{2-4}$

If fetus with mediastinal teratoma survives until birth, tumour compression on mediastinal structures usually manifests with severe respiratory distress in newborn. ${ }^{19-13}$ Early surgical excision is important, but outcome depends on several additional factors such as lung hypoplasia, the stage of heart development, tracheomalacia and peri- and 


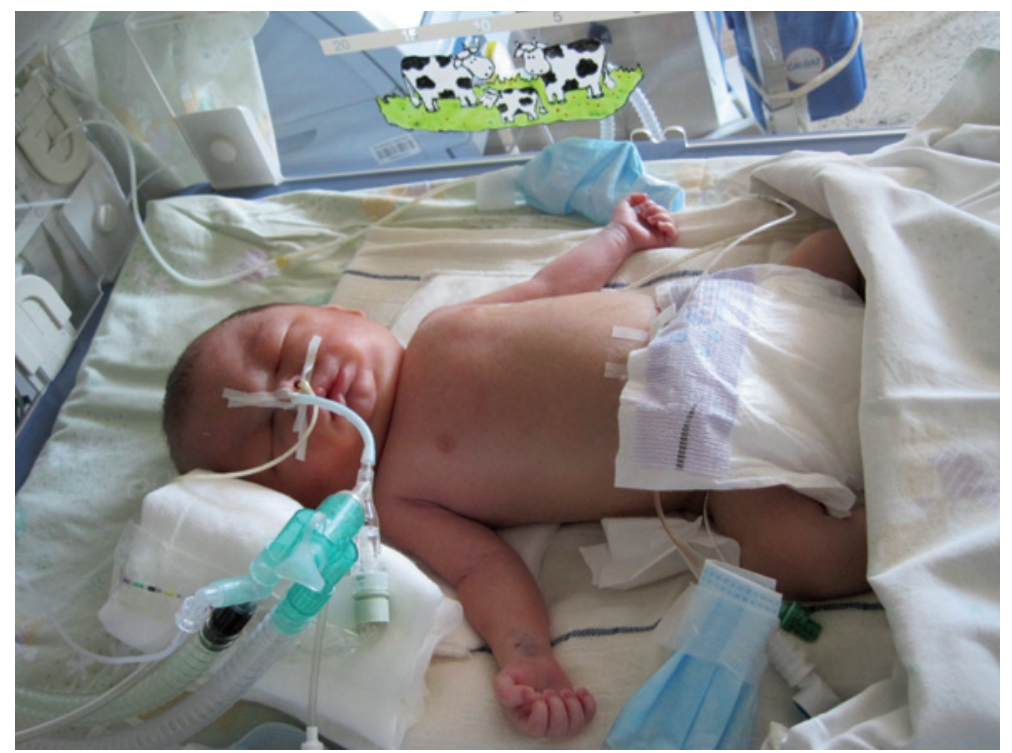

FIGURE 1. Geminus A with severe hydrops fetalis was intubated immediately after birth.

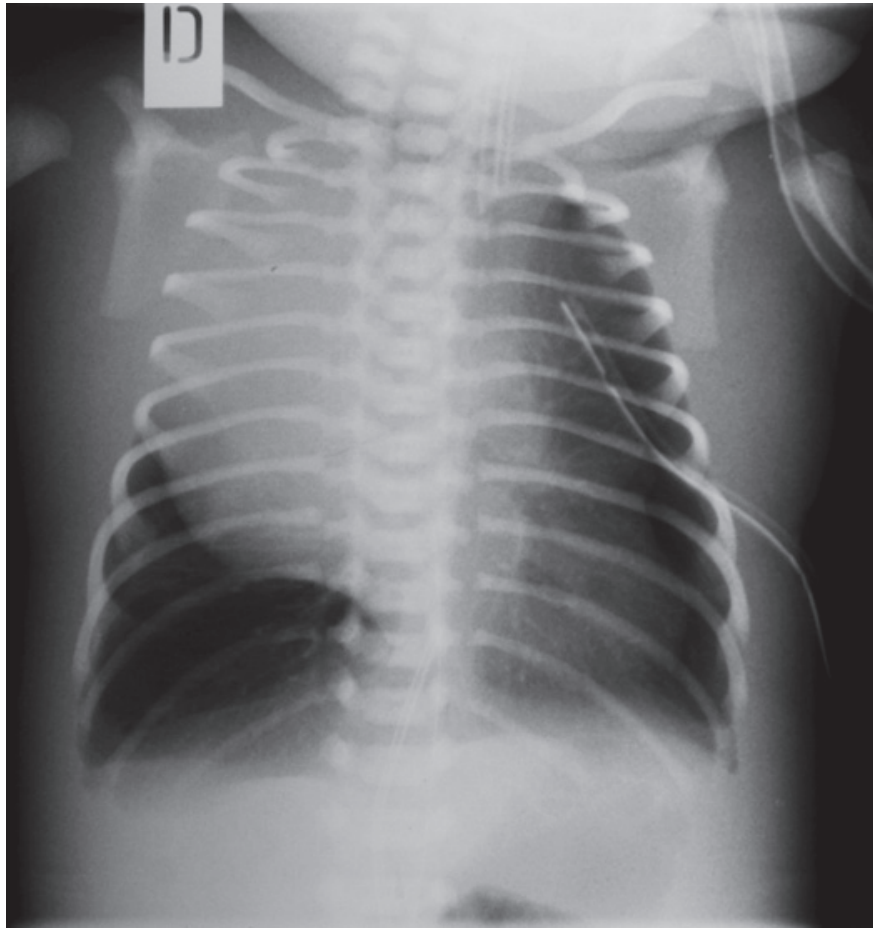

FIGURE 2. Chest $x$-ray after birth demonstrates large round tumour in the right hemithorax. $(6 \times 7 \mathrm{~cm})$ with deviation of mediastinal structures to the left. Soft tissues of the thoracic wall are oedematous.

postoperative complications. . $^{3,6-8,10} \mathrm{We}$ present a case of mediastinal teratoma in a newborn associated with severe NIHF with several complications after birth and surgical procedure which led to the development of chronic respiratory insufficiency.
This is the fifth reported case of a mediastinal teratoma with NIHF in which the newborn survived the neonatal period.

\section{Case report}

A 31-year-old gravida 2 with twins, para 1, was referred to the University Medical Centre Ljubljana because of spontaneous membrane rupture at 32 weeks gestation after an uncomplicated pregnancy. After admission US revealed polyhydramnion and severe NIHF in geminus A. Due to life threatening condition also for healthy geminus B an urgent caesarean section was performed. Geminus A was a boy with Apgar score 3/5 and a birth weight of 3,200 g. He was intubated immediately after birth because of severe NIHF and respiratory distress (Figure 1).

An initial arterial blood gas analysis revealed a respiratory acidosis with a $\mathrm{pH}$ of $6.91, \mathrm{PaCO}_{2} 19$ $\mathrm{kPa}$ and $\mathrm{HCO}_{3}$ of $13 \mathrm{mmol} / \mathrm{l}$. US revealed anterior mediastinal cystic mass extending into the right and left hemithorax with marked pleural effusion bilaterally. Chest $\mathrm{X}$-ray confirmed a homogenous mass in the anterior right hemithorax with shifting of the mediastinum to the left (Figure 2). Ductus arteriosus with left to right shunt was still patent. Because of pleural effusion pleural puncture was performed, further complicated by pneumothorax. Despite ventilation with high frequent oscillations respiratory acidosis still persisted with PCO2 between 10 and $15 \mathrm{kPa}$. Due to low blood pressure (mean arterial pressure $4 \mathrm{kPa}$ ) and very poor urine output he needed vasoactive support with dopamin and dobutamine. US of the heart showed signs of pulmonary artery hypertension. The diagnosis based on radiological finding was congenital cystic adenomatoid malformation, which needed surgical excision on day seven of life.

Right anterolateral thoracotomy was performed with removal of soft policystic encapsulated tumour without invading into the surrounding structures. Tumour was not connected to respiratory system and lungs were macroscopically normally developed. The surgical specimen measured $55 \mathrm{x}$ $50 \mathrm{~mm}$, weighed $54 \mathrm{~g}$, and was extensively sampled.

Microscopically the tumour was predominantly, in $70 \%$, composed by immature tissue derived from different germinal layers. Cystic areas were alternating with solid tissue. The majority of the tumour was characterized by immature neuroectodermal tissue. The elements of mature glial tis- 
sue, ganglion cells and structures of choroid plexus were also randomly interspersed. Mesodermal tissue was represented by mature muscle, bone, adipose tissue, and mature and immature cartilage components with sparsely cellular mesenchymal tissue. The mature components of the tumour were also represented by the microfoci of hepatic differentiation, and pancreatic tissue, as well as sparse elements in the form of skin appendages (Figures 3,4,5). Serum human chorionic gonadotropin and alpha protein were within normal limits, and no further oncologic treatment was necessary.

Hydropic oedemas subsided three days after operation. Urine output and hemodynamic parameters were stable and we discontinued the vasoactive drug support one week after operation. Postoperative course was complicated by a spontaneous pneumothorax on the right side and a sepsis caused by Staphylococcus epidermidis. Fifteen days after operation, the newborn still needed support with synchronized intermittent mandatory ventilation. He had multiple spontaneous episodes of oxygen desaturation. We performed diagnostic rigid bronchoscopy with which we excluded changes in the bronchial system. US of the heart showed signs of pulmonary artery hypertension, persistent ductus arteriosus and patent foramen ovale. We started treatment with nitric oxide and selective inhibitor of phosphodiesterase type 5, with no improvement. Chest X-ray revealed hyperinflation of the left lung and high position of the diaphragm on the right side (Figure 6).

With electromyography we confirmed phrenic nerve palsy and right diaphragm paralysis. At 50 days of age, a second surgery was performed and the right diaphragm was plicated. He was extubated successfully 1 week after plication. After extubation the cyanosis and tachipnoea were periodically observed with paradoxical movement of the chest. Scintigraphy of the lungs revealed smaller right lung without regional perfusion or ventilation defects.

At 3 months of age, he was referred to the intensive care unit with respiratory distress, intubated and mechanically ventilated. Chest $\mathrm{X}$-ray revealed pneumonia and right lung atelectasis. Computed tomography (CT) scan of the lungs again revealed a high position of the right diaphragm and a consolidation of the right and left lung. Due to multiple failures of weaning from mechanical ventilation and extubation one month after admission the third operation was performed with right diaphragm plication and lung biopsy. Biopsy of the

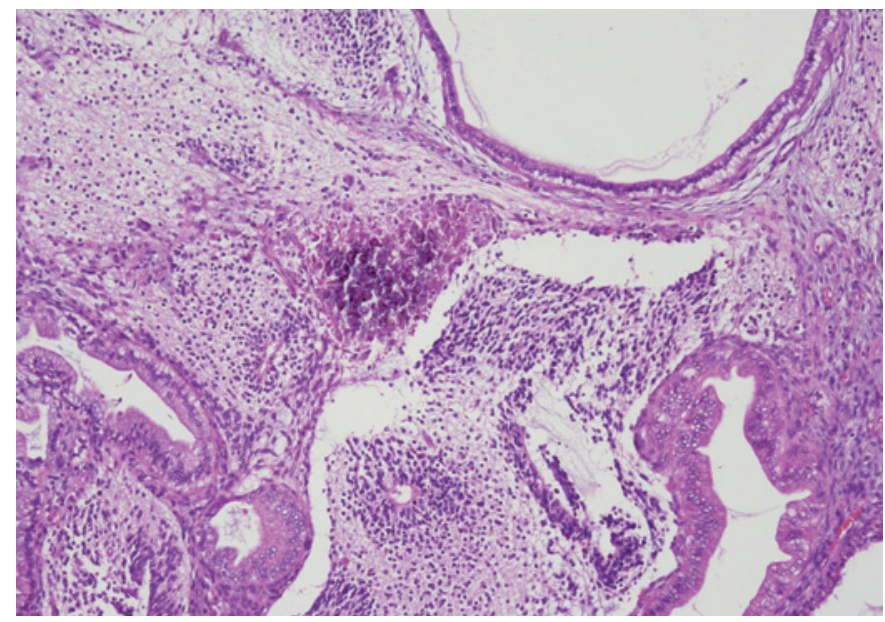

FIGURE 3. Focal calcification and the presence of cystic structures alternating with solid areas composed of mature and immature neural tissue.

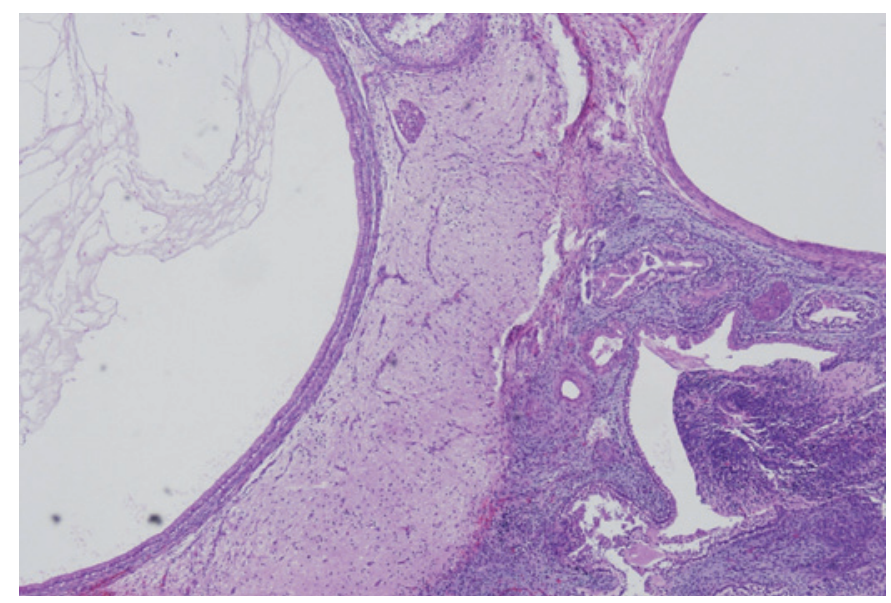

FIGURE 4. Cystic walls lined by columnar and cuboidal epithelium. Solid parts displaying brain tissue comprising of glia cells and primitive neuroepithelium.

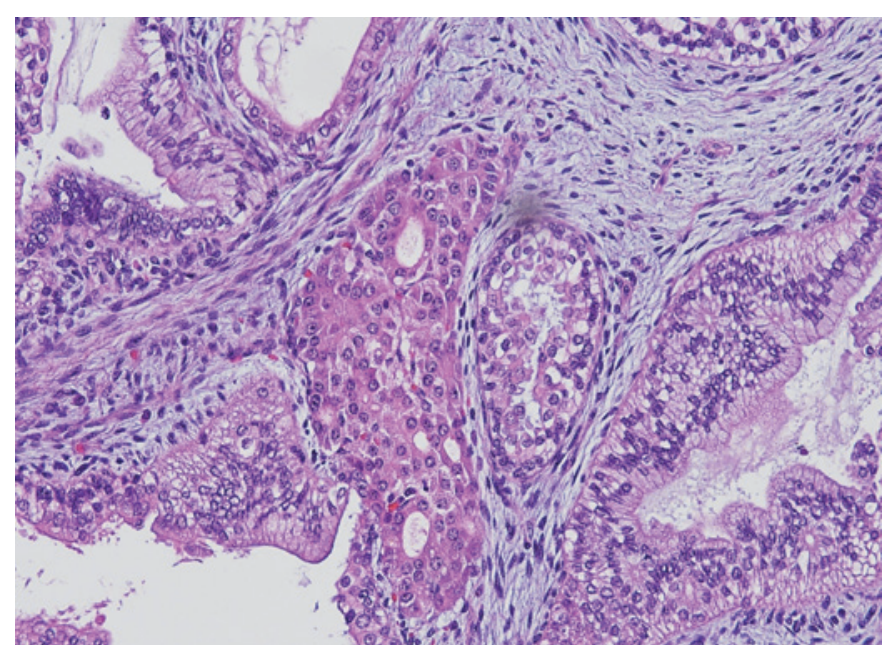

FIGURE 5. Gland-like structure with acinar epithelium resembling pancreatic tissue. Cystic formation embedded in a loose immature stromal tissue. 


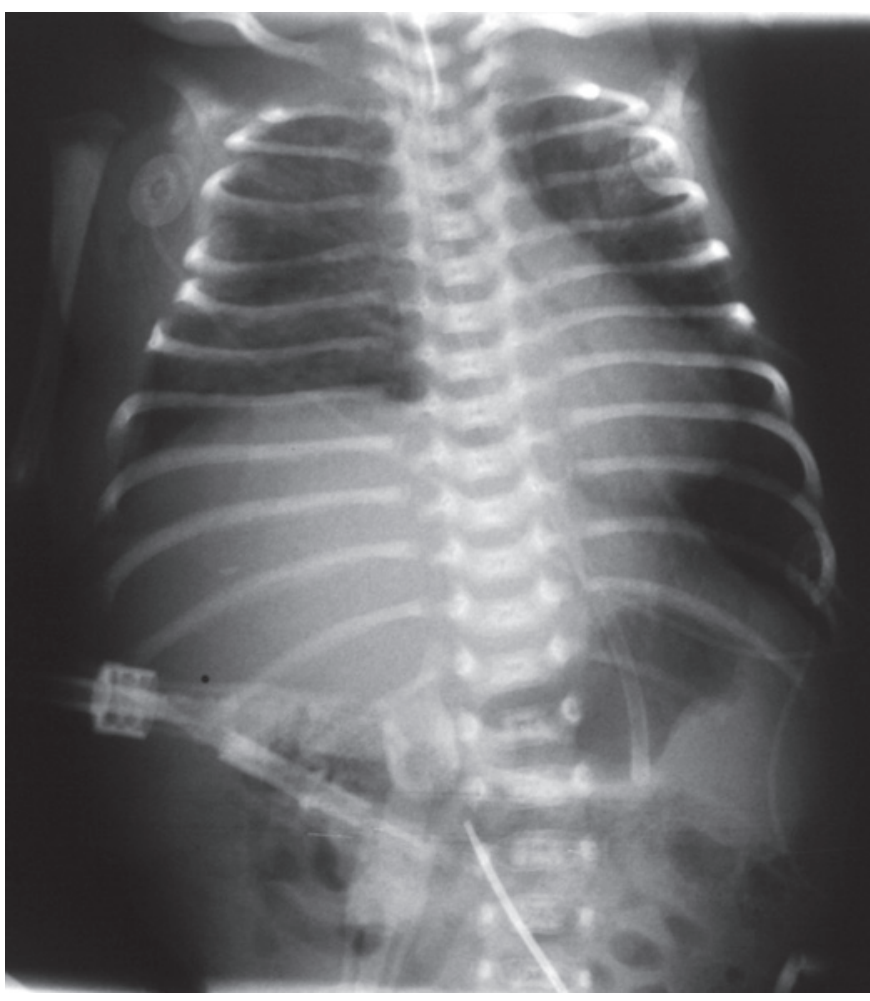

FIGURE 6. Chest x-ray demonstrates high position of the right diaphragm due to postoperative paralysis.

right lower lung lobe revealed acute interstitial pneumonia and alveolitis. After plication and antibiotic treatment, there was clear but slow improvement. He was extubated one month after plication and was well for one month.

At 6 months of age, he was again referred to the intensive care unit with dyspnoea, tachipnoea, cyanosis and severe respiratory acidosis with $\mathrm{PaCO}_{2}$ $10 \mathrm{kPa}$. Bronchoscopy revealed narrowing of the left inferior bronchus. US of the heart showed structurally normal heart with the appropriate function of both ventricles. For further assessment of the cardiovascular function heart catheterization was performed, revealing an abnormal course of both pulmonary arteries with signs of pulmonary artery hypertension. Right middle lung lobe was practically without normal right pulmonary vascularisation. With no improvement in respiratory condition one month after treatment we decided for a tracheostomy. He was breathing with nasal continuous positive airway pressure ventilation (CPAP) on Legendair ventilator (Covidien $\mathrm{AG}^{\mathrm{TM}}$ ) with the addition of oxygen FiO2 0.25-0.35) after the procedure. We disconnected him from the ventilator in order to evoke spontaneous breathing several times per day.
At 11 months of age, paediatric neurologist made a neurologic assessment. He had severe generalized muscle hypotonia, a convergent strabismus and normal proprioceptive reflexes. Babinski sign was still present. Magnetic resonance imaging (MRI) confirmed atrophic frontal brain changes. We excluded metabolic diseases.

At 1 year of age, the boy was discharged from hospital in a stable clinical respiratory condition with tracheostomy and Legendair ventilator. At the age of 2 years, bronchoscopy revealed massive granulation in trachea just below the tracheostoma. After removal of the granulation the tracheostoma was closed and he is breathing on his own.

\section{Discussion}

Mediastinal tumours can be detected before birth by routine antenatal US, most frequently during second and third trimester. With an US we can determine the location of the tumour, pressure on mediastinal structures, cystic or solid tumour components, development of NIHF and polyhydramnion. ${ }^{14}$ With fetal MRI we can obtain more precise information about the tumour and its relationship to adjacent structures. ${ }^{2,4,14}$ Neonatal outcome depends on the size and location of the tumour, presence of NIHF and early prenatal or perinatal intervention. ${ }^{12-4,13}$

Liang et al. evaluated the influence of intrathoracic mass on fetal hemodynamics with Doppler flow velocimetry. They showed in their case that the main changes occurred in the heart and great vessels. ${ }^{5} \mathrm{On}$ the other hand NIHF indicates that tumour causes intrauterine pressure on the heart and major blood vessels. ${ }^{7}$ It is expressed with oedema, ascites, pleural effusion, and hepatomegaly., ${ }^{2,3}$ NIHF and polyhydramnion are poor prognostic signs. ${ }^{3}$ Pressure in the thorax leads to the development of heart dysplasia and lung hypoplasia with reported death prenatally and after birth. ${ }^{7,8,15,16}$ Polyhydramnion develops due to pressure on the oesophagus and decreased swallowing of amniotic fluid. ${ }^{17}$

We found only four reported cases with prenatal identified NIHF caused by mediastinal teratoma, who survived the neonatal period (Table 1). ${ }^{2-4}$ In these cases different approaches were used for a successful outcome. In the case by Takayasu et al. NIHF resolved immediately after aspiration of the tumour cyst. ${ }^{4}$ Merchant et al. recommend in utero resection of tumour if NIHF develops before 30 weeks gestation. After 30 weeks gestation it is important to evaluate lung development and airway 
TABLE 1. Clinical neonatal successfull outcome of fetal mediastinal teratomas with hydrops fetalis

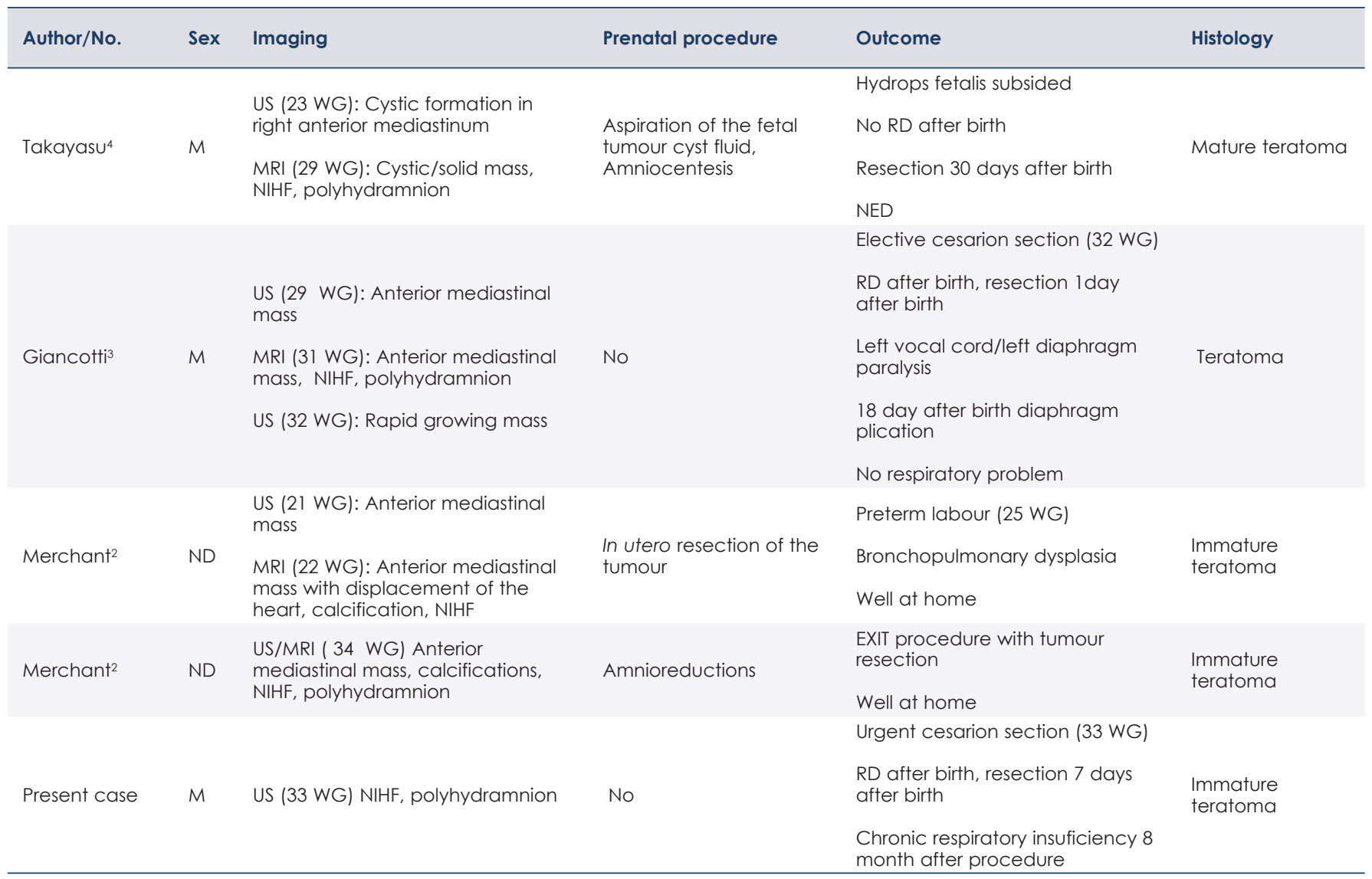

$M=$ male; $M R I=$ magnetic resonance imaging; $N D=$ no data; $N E D=$ no evidence of disease; $N I H F b=$ non-immune hydrops fetalis; RD = respiratory distress; US = ultrasound; WG = weeks gestation

compromise. If the child's airway is compromised we can use ex-utero intrapartum treatment (EXIT procedure) to establish ventilation. ${ }^{2}$ Giancotti et al. reported a prenatally discovered mediastinal tumour with NIHF and resection one day after birth. ${ }^{3}$ In our case regular US were normal up to 30 weeks gestation. After spontaneous membrane rupture in 32 weeks gestation severe NIHF in geminus A and polyhydramnion were discovered. This indicates that tumour growth was fast with severe pressure on mediastinal structures.

Mediastinal teratomas without NIHF that were not discovered prenatally presented with severe respiratory distress in a live newborn after birth. In all cases surgery was performed with removal of the tumour, and mortality in this group was low. ${ }^{9-13}$

Postoperative course was complicated with severe tracheomalacia, wound sepsis and chyle leak in the Mogilner et al. case. ${ }^{10}$ Some authors described diaphragm paralysis with necessary plication after surgery. 3,6 We found only one case in lit- erature with prenatal collapse of the left bronchial system and tracheostomy after surgery at the age of 5 weeks. ${ }^{6}$

Seo et al. reported support with extracorporeal membrane oxygenation after surgery, but in this case a congenital cystic adenomatoid malformation, stocker type III (stocker-III CCAM) was later confirmed and the child had profound persistent fetal circulation postoperatively. Other authors do not mention using extracorporeal membrane oxygenation in children with mediastinal teratoma. In our case, the child had in addition to anatomical changes in the pulmonary vessels several risk factors for the development of chronic respiratory insufficiency including diaphragm paralysis, prematurity and several respiratory infections.

Heart catheterization in our case showed that tumour compression in prenatal period had an impact on the anatomy of mediastinal organs.

In conclusion, it is important to decide for early intervention if NIHF develops. Advances in ra- 
diologic imaging prenatally and after birth are the main tools for diagnostic evaluation of the disease. In cases when NIHF develops the outcome is mainly unpredictable because short and long term sequelae of combined effects of NIHF and teratoma pressure on neighboring organs may develop as we presented with our case.

\section{References}

1. Barksdale E, Obokhare I. Teratomas in infants and children. Curr Opin Pediatr 2009; 21: 344-49.

2. Merchant AM, Hedrick HL, Johnson MP, Wilson RD, Crombleholme TM, Howell $\sqcup$, et al. Management of fetal mediastinal teratoma. J Pediatr Surg 2005; 40: 228-31.

3. Giancotti A, La Torre R, Bevilacqua E, D’Ambrosio V, Pasquali G, Panici PB. Mediastinal masses: a case of fetal teratoma and literature review. Clin Exp Obstet Gynecol. 2012; 39: 384-7.

4. Takayasu H, Kitano Y, Kuroda T, Kuroda T, Morikawa N, Tanaka H, et. al. Successful management of a large fetal mediastinal teratoma complicated by hydrops fetalis. J Pediatr Surg 2012; 45(12): e21-4

5. Liang R, Wang P, Chang FM, Chang $\mathrm{CH}$, Yu CH. Prenatal sonographic characteristics and Doppler blood flow study in a case of a large fetal mediastinal teratoma. Ultrasound Obstet Gynecol 1998; 11: 214-8.

6. Dumbell HR, Coleman AC, Pufidin JM, Winship WS. Prenatal ultrasonographic diagnosis and successful management of mediastinal teratoma, $A$ case report. S Afr Med J 1990; 78: 481-3.

7. Froberg MK, Brown RE, Maylock J, Poling E. In utero development of a mediastinal teratoma: a second-trimester event. Prenat Diagn 1994; 14: 884-7.

8. Aksoy F, Sen C, Danisment N. Congenital mediastinal immature teratoma: a case report with autopsy findings. Turk J Pediatr 2002; 44: 76-9.

9. Kuroiwa M, Suzuki N, Takahashi A, Ikeda H, Hatekeyama S, Matsuyama S, et al. Life- threatening mediastinal teratoma in a neonate. Pediatr Surg Int 2001, 17: 235-8.

10. Mogilner JG, Fonseca J, Davies MR. Life-threatening respiratory distress caused by a mediastinal teratoma in a newborn. J Pediatr Surg 1992; 27: $1519-20$.

11. Takrouri MS, Al-Qahtani A, Ali AM, Al Shakweer W, Kalou MM, Radwan SM Management of neonatal massive anterior mediastinal teratoma. A case report. Middle East J Anesthesiol 2009; 20: 461-4.

12. Lakhoo K, Boyle M, Drake DP. Mediastinal teratomas: rewiev of 15 pediatric cases. J Pediatr Surg 1993; 28: 1161-4.

13. Kreller-Laugwitz $G$, Kobel $H$, Opermann HF. [Mediastinal teratoma in a newborn infant]. [German]. Monatsschr Kinderheilkd 1988; 136: 270-2.

14. Avni FE, Massez A, Cassart M. Tumours of the fetal body: a review. Pediatr Radiol 2009; 39: 1147-57.

15. Noreen S, Heller DS, Faye-Petersen O. Mediastinal teratoma as a rare cause of hydrops fetalis and death: report of 3 cases. J Reprod Med 2008; 53: 708-10.

16. Kuller JA, Laifer SA, Martin JG, MacPherson TA, Mitre B, et al. Unusual presentation of fetal teratoma. J Perinatol 1991; 11: 294-6.

17. Seo $T$, Ando $H$, Watanabe $\mathrm{Y}$, Harada $\mathrm{T}$, Ito F, Kaneko $\mathrm{K}$, et al. Acute respiratory failure associated with intrathoracic masses in neonates. J Pediatr Surg 1999; 34: 1633-7. 